EPJ Web of Conferences 62, 03005 (2013)

DOI: $10.1051 /$ epjconf/20136203005

(C) Owned by the authors, published by EDP Sciences, 2013

\title{
The investigation of properties of short-lived SF isotopes $(Z>100)$ at the focal plane of VASSILISSA separator
}

\author{
Alexandr Svirikhin ${ }^{1}$, Alexander Andreev ${ }^{2}$, Victor Dushin ${ }^{3}$, Maxim Chelnokov ${ }^{1}$, Victor Chepigin ${ }^{1}$, \\ Mohini Gupta ${ }^{4}$, Andrey Isaev ${ }^{1}$, Igor Izosimov ${ }^{1}$, Denis Katrasev ${ }^{1}$, Alexey Kuznetsov ${ }^{1}$, Oleg Malyshev ${ }^{1}$, \\ Ani Minkova ${ }^{5}$, Simon Mullins ${ }^{6}$, Andrey Popeko ${ }^{1}$, Evgeniy Sokol $^{1}$, and Alexander Yeremin ${ }^{1}$ \\ ${ }^{1}$ Flerov Laboratory of Nuclear Reactions, JINR, 141980 Dubna, Russia \\ ${ }^{2}$ Bogoliubov Laboratory of Theoretical Physics, JINR, 141980 Dubna, Russia \\ ${ }^{3}$ V.G. Khlopin Radium Institute, 194021 St. Petersburg, Russia \\ ${ }^{4}$ Manipal University, Madhav Nagar, Manipal, 576104 Karnataka, India \\ ${ }^{5}$ Institute for Nuclear Research and Nuclear Energy, 1784 Sofia, Bulgaria \\ ${ }^{6}$ Laboratory for Accelerator-Based Sciences, 7129 Somerset West, South Africa
}

\begin{abstract}
For experiments aimed at the study of spontaneous fission of transfermium nuclei improvements in the focal plane detector system of recoil separator VASSILISSA have been made. A neutron detector consisting of $54{ }^{3} \mathrm{He}$-filled counters has been mounted around the focal-plane detector chamber. The reaction ${ }^{48} \mathrm{Ca}+{ }^{206} \mathrm{~Pb}=2 \mathrm{n}+{ }^{252}$ No is used for tuning the separator settings and calibrating the detector system with the spontaneous fission of the ${ }^{252}$ No. The average neutron number per ${ }^{252}$ No spontaneous fission event is as large as $\bar{v}=4.06 \pm 0.12$. The short-lived heavy isotopes ${ }^{244,246} \mathrm{Fm}$, produced in the complete fusion reactions ${ }^{40} \mathrm{Ar}+{ }^{206,208} \mathrm{~Pb}$, are investigated. The average number of neutrons per spontaneous fission of ${ }^{244,246} \mathrm{Fm}$ from the experimental data were $(\bar{v}=3.3 \pm 0.3)$ and $(\bar{v}=3.55 \pm 0.50)$, respectively. Both values are determined for the first time.
\end{abstract}

\section{Introduction}

Presently, the available experimental information on the spontaneous fission of transfermium elements mainly con-cerns partial half-lives. For Fm and No isotopes and for a few $\mathrm{Md}$, Lr and $\mathrm{Rf}$ isotopes the total kinetic energy (TKE) and mass distributions of fission fragments from spontaneous fission were also accurately measured [1]. It should be noted, that the investigation of the neutron emission accompanying spontaneous fission (SF) has highlighted importance of dynamics and dissipation for fission processes. The number of neutrons emitted during fission directly depends on the degree of excitation of fission fragments and thus plays an important role in the restoration of the reaction energy balance and aids in exploration of the nuclear properties. On the other hand, the mean number of neutrons per spontaneous fission is a unique characteristic of the nucleus.

The currently available experimental information on a multiplicity distribution of prompt neutrons emitted from spontaneous fission have been obtained for long-lived isotopes using chemical separation

This is an Open Access article distributed under the terms of the Creative Commons Attribution License 2.0, which permits unrestricted use, distribution, and reproduction in any medium, provided the original work is properly cited. 


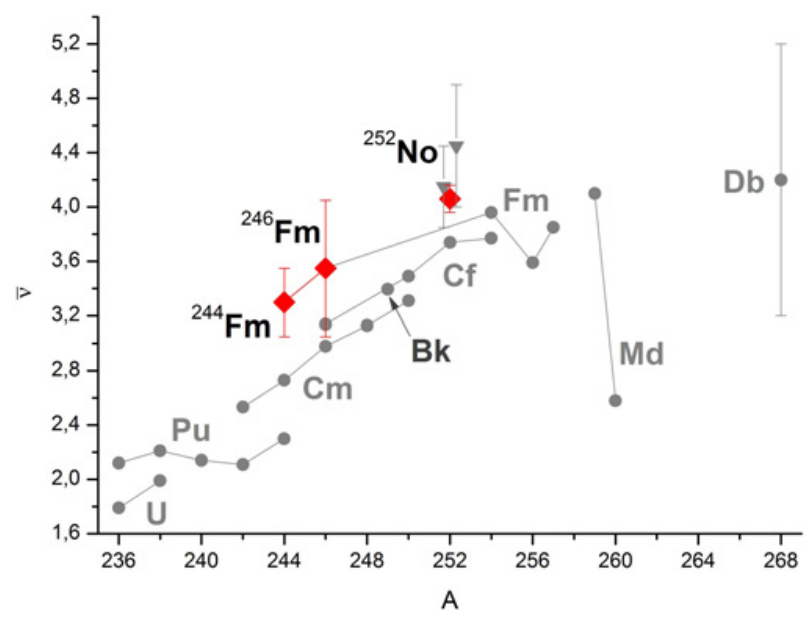

Figure 1. The average number of neutrons per fission as a function of the atomic number A. The latest results measured at VASSILISSA are shown as "diamonds".

techniques [see ref. [1] and references therein] and mechanical evaporation residue (ER) transportation systems [2]. These measurements were performed for elements with $\mathrm{Z}<100$, several neutron-rich Fm isotopes [1] and the isotope ${ }^{252}$ No [2] (see Fig. 1). The experimental set ups and techniques used in those experiments had critical limitations in the half-life measurements, and background conditions due to rather low suppression factors of unwanted reaction products.

The in-flight recoil separator VASSILISSA [3] is widely used for the synthesis and study of decay properties of transfermium nuclei. It is characterized by a high level of suppression of beam particles and unwanted reaction products and high production rates in the region of charge and mass of target nuclei. The transportation time of ER to the focal plane detector [4] is a few microseconds which allowing the investigation of very short-lived isotopes.

\section{Experimental details}

The beam of heavy ions $\left({ }^{48} \mathrm{Ca}\right.$ and ${ }^{40} \mathrm{Ar}$ ) was delivered by the U400 cyclotron of the FLNR (JINR, Dubna). The intensity of the beam passed through the separator target was typically $(2-3) \times 10^{12} \mathrm{~s}^{-1}$ for both types of accelerated particles. In the both experiments the enriched lead material was used for the target. The target segments were mounted on a rotating target wheel in order to withstand the intense beam.

The focal-plane semiconducting Si detector system of VASSILISSA separator allows measuring the energy of both fission fragments from the spontaneous fission of the implanted ER. The detector array consists of five identical 16-strip silicon wafers (see Fig. 2). The active area of a single silicon strip detector is $60 \times 60 \mathrm{~mm}^{2}$. Each of its strips is position sensitive in the vertical direction with a position resolution of $0.5 \mathrm{~mm}$ (FWHM) for sequential $\alpha-\alpha$ decays, $0.8 \mathrm{~mm}$ for ER- $\alpha$, and $1.0 \mathrm{~mm}$ for ER-SF events. The average energy resolution is about $25 \mathrm{keV}$ for the standard $\alpha$-source. Four wafers are mounted in the backward hemisphere facing the stop detector. They measure escaping alphas or fission fragments, and the total geometrical efficiency is $85 \%$ of $4 \pi$.

To study spontaneous fission of heavy short-lived isotopes in more detail, a neutron detector consisting of $54{ }^{3} \mathrm{He}$-filled (4 Atm) counters was mounted around the focal-plane detector chamber of VASSILISSA separator. The focal-plane detector assembly was housed in a cylindrical vacuum chamber $120 \mathrm{~mm}$ in diameter. Neutron counters were placed around this chamber in three concentric 


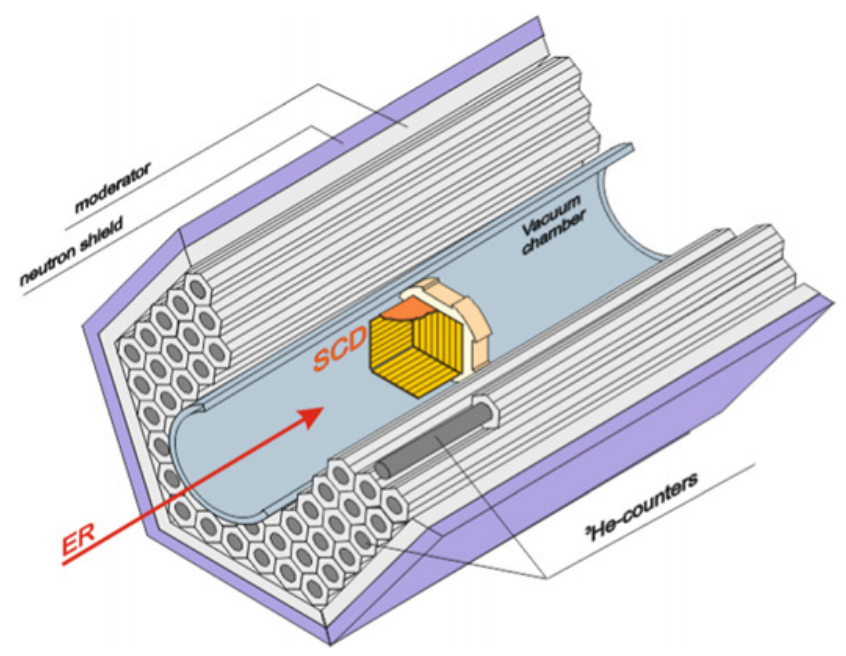

Figure 2. Schematic view of focal plane detector surrounded by neutron counters and moderator.

rings (see Fig. 2). From the outside, the neutron counters were covered by plates of plexiglass and boron polyethylene, both $5 \mathrm{~cm}$ thick, to slow down and capture background neutrons from the outside of the neutron counter. It allowed us to reduce the neutron background by one order of magnitude. The electronic system developed for these prompt neutron multiplicity measurements was started by the pulses from the SF amplifier outputs. A special module collected all signals from neutron counters. SF-like signals started the timing counter module. After the START signal all events from the neutron counters within the time period of $128 \mu$ s were stored in a data acquisition system as unique "Neutron" events. Those events were used for creating spectra of the experimental multiplicity distribution.

To measure the efficiency of detection of one neutron and better simulate the experimental condition, the thin aluminum foil $(1.2 \mu \mathrm{m})$ with small quantity of curium chloride was placed inside the focal-plane detector (the intensity of the source was 3-4 SF events per second). The neutron detector efficiency measured using the ${ }^{248} \mathrm{Cm}$ source was $40 \pm 1 \%$. Though the beam intensity was high, the background conditions remained favorable during the whole experiment: the background neutron counting in the detector room was 80 random counts per second or less.

\section{Experimental results}

In the last years we carried out several experiments aimed to investigate properties of short-lived SF isotopes.

Heavy-ion fusion reactions ${ }^{48} \mathrm{Ca}+{ }^{204} \mathrm{~Pb}$ and ${ }^{44} \mathrm{Ca}+{ }^{208} \mathrm{~Pb}$ leading to the same compound nucleus ${ }^{252}$ No were run in attempts to produce new neutron-deficient spontaneous-fission isotopes of ${ }^{249,250}$ No using the electrostatic separator VASSILISSA in the year 2002 [5]. Production cross-sections for the spontaneous fission activities with the half-lives 5.6 and $54 \mu$ s observed in these reactions are compared with the measured ones for the well-known isotopes of ${ }^{251-255}$ No formed in the heavy-ion fusion reactions ${ }^{48} \mathrm{Ca}+{ }^{206} \mathrm{~Pb}$ and ${ }^{48} \mathrm{Ca}+{ }^{208} \mathrm{~Pb}$. In the year 2004 for experiments aimed at the study of spontaneous fission of transfermium nuclei improvements in the focal plane detector system of recoil separator VASSILISSA have been made. The first edition of neutron detector consisting of $72^{3} \mathrm{He}$ filled counters has been mounted around the focal plane detector chamber (neutron detection efficiency was 
$25 \%$ ) [6]. A multiplicity distribution of prompt neutrons emitted in spontaneous fission of ${ }^{252} \mathrm{No}$, formed in the reaction ${ }^{48} \mathrm{Ca}+{ }^{206} \mathrm{~Pb}$, was measured in test experiments and was equal to $\bar{v}=4.43 \pm 0.45$. This value is in good agreement with that from literature $(\bar{v}=4.15 \pm 0.30)$ [2].

After modernizing the detection system and installing new neutron detector consisting of $54{ }^{3} \mathrm{He}-$ filled counters (Fig. 2) the series of experiments aimed at the study of spontaneous fission of transfermium nuclei were carry out.

The neutron-deficient isotope ${ }^{246} \mathrm{Fm}$, produced in the complete fusion reaction ${ }^{40} \mathrm{Ar}+{ }^{208} \mathrm{~Pb}$, was investigated in the year 2008. The half-life was determined to be as $\mathrm{T}_{1 / 2}=(1.3 \pm 0.2) \mathrm{s}$. This agrees with the earlier reported value: $1.2 \mathrm{~s}$ [7]. The SF branching ratio for ${ }^{246} \mathrm{Fm}$ was estimated to be $b_{\mathrm{SF}}=(5 \pm 3) \%$ which is comparable with the values obtained earlier: $8 \%$ [7]. The main goal of the experiment was to determine the neutron multiplicity at spontaneous fission of this isotope. From the experimental data the average number of neutrons per spontaneous fission of ${ }^{246} \mathrm{Fm}$ was determined for the first time $(\bar{v}=3.55 \pm 0.50)[8]$.

In the year 2010, we carried out an experiment [9] aimed at investigating the properties of spontaneous fission of neutron deficient isotopes of ${ }^{252} \mathrm{No}$ and ${ }^{244} \mathrm{Fm}$ produced in the reaction with ${ }^{48} \mathrm{Ca}$, ${ }^{40} \mathrm{Ar}$-beams and ${ }^{206} \mathrm{~Pb}$-target. In the first experiment, 1700 events of ${ }^{252} \mathrm{No}$ spontaneous fission were detected over a $24 \mathrm{~h}$ exposure, which allowed us to measure the multiplicity distributions of neutrons with a high degree of accuracy and to test operation of the entire detector system. The average number of neutrons per one SF of ${ }^{252}$ No was determined to be $\bar{v}=4.06 \pm 0.12$. The determined value is in good agreement with the earlier results $[2,6]$.

The next experiment was done to study the properties of ${ }^{244} \mathrm{Fm}$ formed in the $2 n$ channel of the complete fusion reaction ${ }^{40} \mathrm{Ar}+{ }^{206} \mathrm{~Pb}$. The cross section of the recoil nucleus production reaction is about $3 \mathrm{nb}\left(\mathrm{E}_{1 / 2}=186 \mathrm{MeV}\right)$. The experiment lasted for 7 days, 212 events pertaining to ${ }^{244} \mathrm{Fm}$ spontaneous fission were registered. By analysis of the correlations between ER and SF events, the halflife of the ${ }^{244} \mathrm{Fm}$ was determined to be $\mathrm{T}_{1 / 2}=(3.47 \pm 0.26) \mathrm{ms}$. This agrees with the earlier reported value $3.12 \pm 0.08$ [10]. For the ${ }^{244} \mathrm{Fm}$ nucleus, the pre-neutron total kinetic energy of the fragments was assessed to be approximately (198 \pm 15$) \mathrm{MeV}$. It closely agrees with the previously published data [10]. This value was calculated by adding up the amplitudes of the signals in the focal-plane and the side detectors and was determined with allowance for the calibration data gained from the ${ }^{252}$ No experiment. No $\alpha$ decay which could be assigned to the ${ }^{244} \mathrm{Fm}$ nucleus decay was detected.The average number of neutrons was determined as $\bar{v}=3.3 \pm 0.3$. The systematic of average neutron numbers per SF is presented in the Figure 1.

We propose to undertake a comprehensive study of SF in heavy short lived neutron deficient isotopes is planned including in the lighter $\mathrm{Cf}$-No region. In the near future, these experiments can be carried out at Dubna after the modernization of VASSILISSA.

Works on VASSILISSA setup was performed partially under the financial support of the Russian Foundation for Basic Research (contract 13-02-12003-ofi-m-2013) and JINR grant No. 13-902-07.

\section{References}

[1] D.C. Hoffman, Nucl. Phys. A 502, 22 (1989)

[2] Yu.A. Lazarev et al., Phys. Lett. B 52, 321 (1974)

[3] A.V. Yeremin et al., Phys. At. Nucl. 66, 1042 (2003)

[4] O.N. Malyshev et al., Nucl. Instr. Meth. A 440, 86 (2000)

[5] A.V. Belozerov et. al,. Eur. Phys. J. A 16, 447 (2003) 
[6] A.V. Yeremin et al., Nucl. Instr. Meth. A 539, 441 (2005)

[7] D.C. Hoffman et al., Phys. Rev. C 22, 1581 (1980)

[8] A.I. Svirikhin et al., Eur. Phys. J. A 44, 393 (2010)

[9] A.I. Svirikhin et al., Eur. Phys. J. A 48, 121 (2012)

[10] J. Khuyagbaatar et al., Eur. Phys. J. A 37, 177 (2008) 